\title{
Profilaxia Pós-Exposição (PEP) como modelo de prevenção combinada: análise do perfil epidemiológico dos usuários em um munícipio do estado de Minas Gerais
}

\author{
Post-Exposure Prophylaxis (PEP) as a combined prevention model: analysis of the \\ epidemiological profile of users in a municipality in the state of Minas Gerais
}

La profilaxis posexposición (PEP) como modelo de prevención combinado: análisis del perfil epidemiológico de los usuarios en un municipio del estado de Minas Gerais

Lucas Guilhermino dos Santos ${ }^{1 *}$, Isadora Martins e Campos ${ }^{1}$, Marcos de Assis Moura ${ }^{1,2}$.

\section{RESUMO}

Objetivo: Descrever o perfil epidemiológico dos usuários da PEP em um munícipio do estado de Minas Gerais. Métodos: Trata-se de análise dos prontuários de pacientes que utilizaram o PEP como medida de prevenção à contaminação após exposição sexual consensual no serviço de atendimento especializado (SAE) de um munícipio do estado de Minas Gerais no período de Janeiro de 2015 até Julho de 2017, considerando as seguintes variáveis como importância para o estudo: sexo, estado civil, ocupação, escolaridade, uso de drogas ou álcool, DST prévia, prática sexual, conhecimento sobre o risco com o parceiro (sorologia) . Resultados: O perfil epidemiológico verificado corresponde à prevalência de usuários do sexo masculino, totalizando $75 \%$, o que corrobora a ideia de tendência dos mesmos a comportamentos de maior risco. Já em relação ao estado civil, observou-se o predomínio importante de "solteiros", levando à conclusão de que há um déficit relevante no uso de métodos contraceptivos de barreira $O$ risco do parceiro era desconhecido por mais de $90 \%$ das pessoas, criando-se a hipótese de que as relações foram eventuais. Conclusão: Conclui-se que os participantes deste estudo possuem conhecimento inadequado sobre os benefícios da profilaxia, dificultando a adesão, tal como foi traçado o perfil epidemiológico.

Palavras-chave: HIV, Prevenção de doenças, Profilaxia pós-exposição.

\begin{abstract}
Objective: To describe the epidemiological profile of PEP users in a city in Minas Gerais. Methods: This is an analysis of the medical records of patients who used the PEP as a measure to prevent contamination after consensual sexual exposure in the specialized care service (SAE) in a city in Minas Gerais, from January 2015 to July 2017, considering the following variables as importance for the study: sex, marital status, occupation, education, use of drugs or alcohol, previous STD, sexual practice, knowledge about risk with the partner (serology). Results: The epidemiological profile verified corresponds to the prevalence of male users, totaling $75 \%$, which corroborates the idea of their tendency towards higher risk behaviors. Regarding marital status, an important predominance of "singles" was observed, leading to the conclusion that there is a relevant deficit in the use of barrier contraceptive methods. The risk of the partner was unknown by more than $90 \%$ of people, creating the hypothesis that the relationships were eventual. Conclusion: It is concluded that the participants of this study have inadequate knowledge about the benefits of prophylaxis, making it difficult to adhere to it, just as the epidemiological profile was traced.
\end{abstract}

Keyword: HIV, Disease prevention, Post-exposure prophylaxis.

\footnotetext{
${ }_{1}^{1}$ Faculdade de Ciências Médicas e da Saúde de Juiz de Fora (Suprema), Juiz de Fora - MG.

${ }^{*}$ E-mail: lucasguilherminos@gmail.com

${ }^{2}$ Universidade Federal de Juiz de Fora (UFJF), Juiz de Fora - MG.
} 


\section{RESUMEN}

Objetivo: Describir el perfil epidemiológico de los usuarios de PEP en una ciudad de Minas Gerais. Métodos: Este es un análisis de los registros médicos de pacientes que utilizaron la PEP como medida para prevenir la contaminación después de la exposición sexual consensuada en el servicio de atención especializada (SAE) en una ciudad de Minas Gerais desde enero de 2015 hasta julio 2017, considerando las siguientes variables como importancia para el estudio: sexo, estado civil, ocupación, educación, uso de drogas o alcohol, ETS previas, práctica sexual, conocimiento sobre el riesgo con la pareja (serología). Resultados: El perfil epidemiológico verificado corresponde a la prevalencia de usuarios masculinos, totalizando $75 \%$, lo que corrobora la idea de su tendencia hacia comportamientos de mayor riesgo. Con respecto al estado civil, se observó un importante predominio de los "solteros", lo que llevó a la conclusión de que existe un déficit relevante en el uso de métodos anticonceptivos de barrera. El riesgo de la pareja era desconocido por más del $90 \%$ de las personas, creando La hipótesis de que las relaciones eran eventuales. Conclusión: Se concluye que los participantes de este estudio tienen un conocimiento inadecuado sobre los beneficios de la profilaxis, lo que dificulta su cumplimiento, al igual que se trazó el perfil epidemiológico.

Palabra clave: VIH, Prevención de enfermedades, Profilaxis posexposición.

\section{INTRODUÇÃO}

A síndrome da Imunodeficiência Adquirida (AIDS), com o passar dos anos, vem assumindo um perfil de cronicidade caracterizado pela queda nas taxas de transmissão vertical do vírus da imunodeficiência humana (HIV), redução da morbimortalidade da infecção e o aumento da expectativa e qualidade de vida das pessoas que vivem com HIV (BENN P, et al., 2011).

Segundo Marins JRP, et al. (2003), a quarta década da epidemia de HIV no Brasil engloba importantes dados epidemiológicos, como a curva de infecções, casos de evolução da AIDS e mortes pela mesma, refletindo uma redução drástica da morbimortalidade graças ao uso amplo da terapia antirretroviral.

Os últimos dados epidemiológicos mostram que 73\% dos novos casos de HIV em 2017 ocorreram no sexo masculino. Quando estratificado por orientação sexual autodeclarada, os homens homossexuais e bissexuais são maioria entre as notificações de casos de HIV (46,5\% e 42,8\%, respectivamente); com destaque de uma tendência de crescimento para todas as categorias de orientação sexual entre os homens (BRASIL, 2019).

O PEP se caracteriza como uma urgência médica e, por isso, deve ser iniciado o mais precocemente possível, idealmente nas primeiras 2 horas após a exposição, tendo como limite as 72 horas subsequentes à exposição (CAMPBELL TB, et al., 2012).

A rede de PEP no Brasil deve contar necessariamente com serviços de atendimento 24 horas para a realização do primeiro atendimento e com devido encaminhamento para seguimento clínico por todo período total de até 3 meses (COHEN MS, et al.,2011).

A indicação da PEP é dependente de uma série de fatores, dentre eles: o tipo de material biológico envolvido e de exposição, o tempo transcorrido entre a exposição e o atendimento e a condição sorológica para HIV da pessoa exposta e da pessoa fonte (MAYER KH, et al., 2012; FERNANDEZ SB, et al., 2013).

Ademais, a PEP não é indicada quando a pessoa exposta já se encontra infectada pelo HIV (infecção prévia à exposição) ou quando a infecção pelo HIV pode ser descartada na pessoa fonte ou em casos em que a toxicidade dos medicamentos supere o risco de transmissão do HIV (OTTEN RA, et al., 2000; MAKSUD I, et al., 2015).

Poucos estudos demostram a eficácia e a aplicabilidade da PEP como método preventivo ao HIV/AIDS (MAYER KH, et al., 2012). Sendo a sustentação dessa profilaxia recomendada pela World Health Organization 
(WHO) desde 2014, devido aos resultados positivos em estudos realizados em animais, após exposições ocupacionais em profissionais da saúde, após exposições sexuais não consensuais e por transmissão vertical (OTTEN RA, et al., 2000; ROLAND ME, et al., 2011).

No Brasil, é frequente que as populações-chave mais vulneráveis, como a de homens que fazem sexo com homens $(\mathrm{HSH})$, enfrentem barreiras ou dificuldades de acesso aos serviços de saúde por questões sociais ou econômicas. É comum que haja resistência à testagem sorológica de HIV devido a questões culturais, bem como em comparecer a serviços especializados por medo de discriminação e consequências sociais.

Sendo assim, tais grupos não recebem, muitas vezes, o atendimento recomendado que abranja desde estratégias de prevenção até o tratamento multidimensional, comprometendo intervenções sobre as infecções sexualmente transmissíveis que podem, no futuro, impactar tanto a saúde individual quanto a coletiva (BAGGALEY R, et al., 2015).

Isto posto, um estudo que contemple tal temática, tendo em vista a relevância da PEP como estratégia de prevenção combinado e os seus benefícios, bem como as dificuldades à sua ampla utilização perante a comunidade científica e a sociedade como um todo, foi realizado com objetivo de traçar o perfil epidemiológico dos usuários da PEP em um munícipio do estado de Minas Gerais.

\section{MÉTODOS}

Foi realizado um estudo retrospectivo com a avaliação e análise de 184 prontuários de pacientes que utilizaram o PEP como medida de prevenção à contaminação após exposição sexual consensual no serviço de atendimento especializado (SAE) do departamento de doença sexualmente transmissíveis e AIDS de um munícipio do estado de Minas Gerais, no período de Janeiro de 2015 até Julho de 2017.

Considerando as seguintes variáveis como importância para o estudo: sexo, estado civil, ocupação, escolaridade, deficiência física ou mental, uso de drogas ou álcool, DST prévia, HIV prévio ao PEP , prática sexual, conhecimento sobre o risco com o parceiro (sorologia) e todos exames submetidos pelo SAE.

Os dados relativos a estas análises foram tabulados em planilhas do software Excel e receberam tratamento estatístico, sendo expostos nos resultados do artigo. Foi realizado uma revisão bibliográfica sobre o tema nas principais bases indexadores de acesso aberto, sendo selecionados 22 artigos relevantes ao tema para construir discussão.

O presente projeto de pesquisa enquadra-se em uma natureza descritiva do tipo transversal, tendo em vista que o objetivo é verificar o perfil epidemiológico dos usuários de PEP um munícipio do estado de Minas Gerais. Desta forma, requer apenas uma observação.

O critério de inclusão do presente projeta de pesquisa são indivíduos que utilizaram o PEP no serviço de atendimento especializado (SAE) do departamento de doença sexualmente transmissíveis e AIDS de um munícipio do estado de Minas Gerais no período de janeiro de 2015 até julho de 2017. Os critérios de exclusão do presente projeto de pesquisa são menores de 15 anos, indivíduos vítimas de violência sexual e outros usos inadequados da estratégia de prevenção.

Os riscos são a perda e exposição dos dados coletados. Os benefícios são a coleta de dados sobre o conhecimento da população sobre a disponibilidade do PEP, estabelecendo um perfil epidemiológico levando a um aprofundamento sobre o tema e melhora taxas de adesão.

O projeto foi previamente aprovado pelo Comitê de Ética em Pesquisa da sociedade universitária para o ensino médico assistencial LTDA (SUPREMA) (CEP/FCMS-JF) regulamentado pelo Conselho Nacional de Ética em Pesquisa (CONEP) como número 2.720.881.

\section{RESULTADOS}

O perfil epidemiológico verificado corresponde à prevalência de usuários do sexo masculino, totalizando $75 \%$, o que corrobora a ideia de tendência dos mesmos a comportamentos de maior risco. Já em relação ao 
estado civil, observou-se o predomínio importante de "solteiros", levando à conclusão de que há um déficit relevante no uso de métodos contraceptivos de barreira, permitindo grande propagação de várias ISTs e justificando o crescente número de diagnósticos atuais, com destaque para epidemia de sífilis.

Ademais, deve-se destacar ainda a possibilidade de exposição a comportamentos alarmantes, como compartilhamento de seringas de drogas injetáveis por esse público. Tomando tal ideia como partida, faz-se necessário a intensificação de campanhas preventivas de educação em saúde, bem como a popularização da PEP.

Acerca das ocupações mais frequentes entre os usuários da PEP (Tabela 1), os estudantes representam uma parcela importante $(23,9 \%)$ e podem ser relacionados a grupos mais jovens que, apesar de possuírem amplo acesso aos meios de comunicação na maioria dos casos, negligenciam os métodos de barreira. Do mesmo modo, a prevalência de níveis de escolaridade avançados (42,9\% superior e 40,7\% médio) conduzem à conclusão de que, apesar de provavelmente bem instruídos, admitem a mesma vulnerabilidade.

Em relação ao uso de drogas lícitas ou ilícitas, 48,9\% dos entrevistados negaram qualquer vício e 36,9\% relataram uso associado de álcool, o que pode comprometer o estado mental do paciente, levando-o a atitudes menos seguras, sejam elas sexuais ou não.

Houve ainda uma baixa associação com outras ISTs prévias, sendo que $88,5 \%$ do total dos pacientes negou patologia prévia. Contudo, diante da via comum, a falta de proteção predispõe o mesmo público a futuras contaminações, como apresentado por $5 \%$ dos casos que declararam sífilis prévia e 4,3\%, gonorreia.

A prática heterossexual foi declarada por $69,5 \%$ dos indivíduos, enquanto $26,6 \%$ declararam-se bissexuais. Já o tipo sexual prevalente foi vaginal $(71,1 \%)$, seguido por anal $(14,6 \%)$, dos quais a maioria foi receptiva, o que se consolida como uma prática de maior risco de aquisição do HIV (Gráfico 1).

Apenas $17,8 \%$ dos casos disseram fazer uso de métodos de proteção, não podendo fazer distinção se houve falha do mesmo ou eventual não uso. O risco do parceiro era desconhecido por mais de $90 \%$ das pessoas, criando-se a hipótese de que as relações foram eventuais. Apenas $4,8 \%$ dos casos tinham conhecimento de que o parceiro era portador de HIV.

Gráfico 1 - Gráfico comparativo entre os tipos de prática sexual dos pacientes atendidos no SAE.

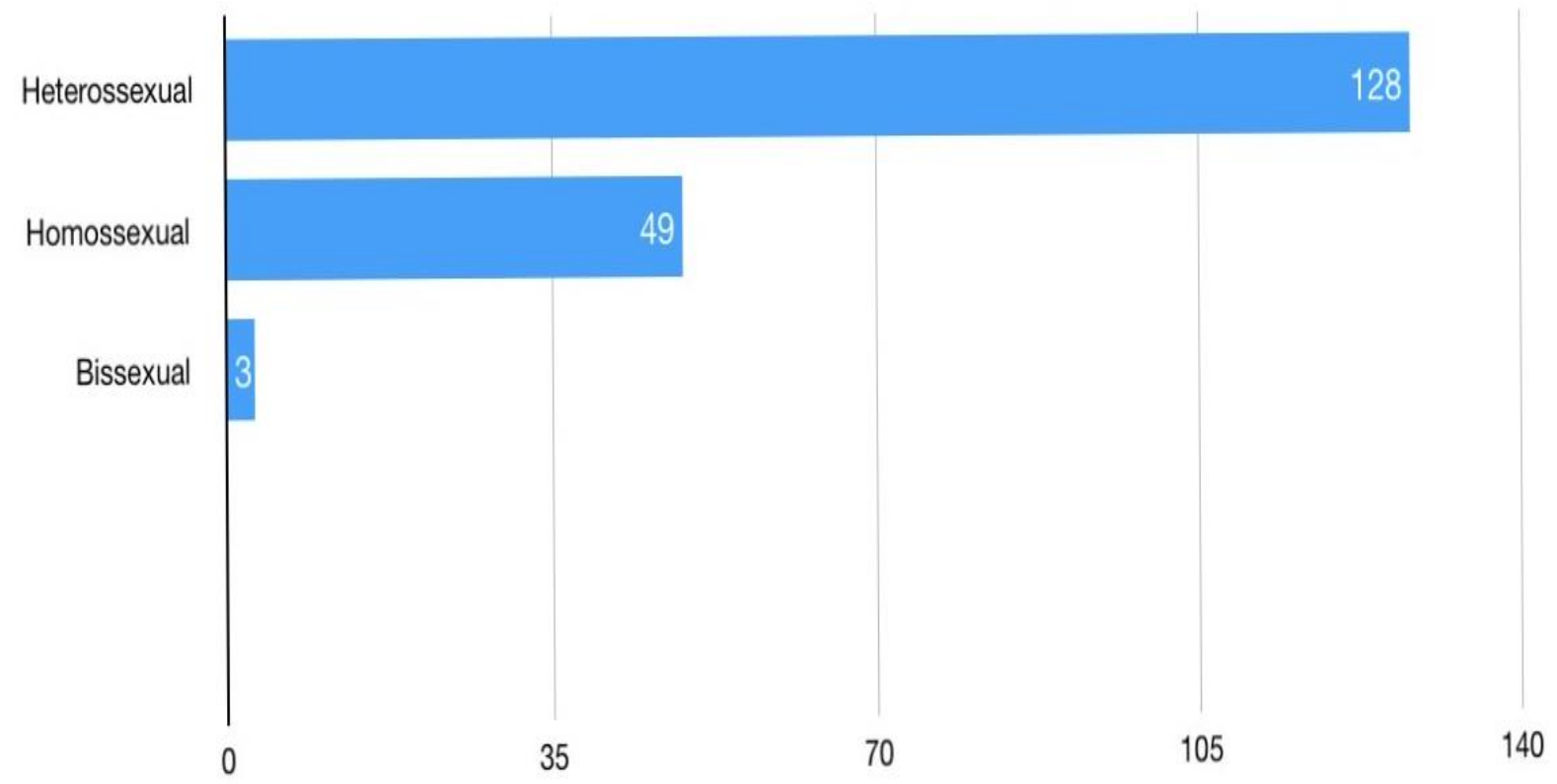

Fonte: Santos LG, et al., 2020. 


\section{Revista Eletrônica Acervo Saúde / Electronic Journal Collection Health | ISSN 2178-2091}

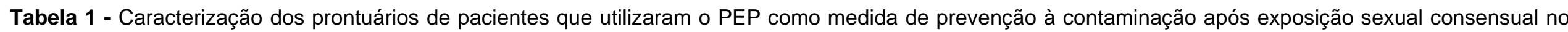

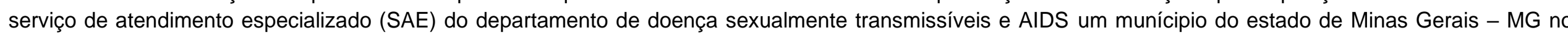
período de Janeiro de 2015 até Julho de 2017.

\begin{tabular}{|c|c|c|}
\hline Variáveis & $n=184$ & $\%$ \\
\hline \multicolumn{3}{|l|}{ Sexo } \\
\hline Feminino & 46 & 25 \\
\hline Masculino & 138 & 75 \\
\hline \multicolumn{3}{|l|}{ Estado Civil } \\
\hline Solteiro & 157 & 85,3 \\
\hline Casado & 20 & 10,8 \\
\hline Divorciado & 7 & 3,8 \\
\hline \multicolumn{3}{|l|}{ Profissão } \\
\hline Estudante & 44 & 23,9 \\
\hline Desempregado & 6 & 3,2 \\
\hline Outros & 134 & 72,8 \\
\hline \multicolumn{3}{|l|}{ Escolaridade } \\
\hline Superior & 79 & 42,9 \\
\hline Médio & 75 & 40,7 \\
\hline Fundamental & 17 & 9,3 \\
\hline Ignorados & 13 & 7 \\
\hline \multicolumn{3}{|c|}{ Uso de drogas ou álcool } \\
\hline Não & 90 & 48,9 \\
\hline Maconha apenas & 14 & 7,6 \\
\hline Álcool & 68 & 36,9 \\
\hline Álcool e cocaína & 12 & 6,5 \\
\hline \multicolumn{3}{|l|}{ DST prévia } \\
\hline Gonorreia & 8 & 4,3 \\
\hline Sífilis & 9 & 4,8 \\
\hline Candidíase & 4 & 2,1 \\
\hline Não & 163 & 88,5 \\
\hline
\end{tabular}

REAS / EJCH | Vol.12(10) | e5098 | DOI: https://doi.org/10.25248/reas.e5098.2020 Página 5 de 10 


\section{Revista Eletrônica Acervo Saúde / Electronic Journal Collection Health | ISSN 2178-2091}

\begin{tabular}{|c|c|c|}
\hline \multicolumn{3}{|l|}{ Prática Sexual } \\
\hline Hétero & 128 & 69,5 \\
\hline Homo & 49 & 26,6 \\
\hline $\mathrm{Bi}$ & 3 & 1,6 \\
\hline Ignorados & 4 & 2,1 \\
\hline \multicolumn{3}{|l|}{ Tipo Sexual } \\
\hline Vaginal & 131 & 71,1 \\
\hline Anal & 27 & 14,6 \\
\hline Oral & 6 & 3,2 \\
\hline Anal Insertivo & 10 & 5,4 \\
\hline Anal Receptivo & 15 & 8,1 \\
\hline Ignorados & 5 & 2,7 \\
\hline \multicolumn{3}{|l|}{ Método Proteção } \\
\hline Não & 140 & 76 \\
\hline Sim & 33 & 17,8 \\
\hline Ignorados & 11 & 5,9 \\
\hline \multicolumn{3}{|l|}{ Risco do Parceiro } \\
\hline Desconhecido & 168 & 91,3 \\
\hline $\mathrm{HIV}+$ & 12 & 6,5 \\
\hline VDRL & 4 & 2,1 \\
\hline \multicolumn{3}{|l|}{ HIV prévio } \\
\hline Ignorados & 169 & 91,8 \\
\hline Não & 6 & 3,2 \\
\hline $\mathrm{HIV}+$ & 9 & 4,8 \\
\hline \multicolumn{3}{|l|}{$\mathrm{HCV}$} \\
\hline NR & 173 & 94 \\
\hline Reagente & 1 & 0,5 \\
\hline Ignorados & 10 & 5,5 \\
\hline
\end{tabular}

Fonte: Santos LG, et al., 2020

REAS / EJCH | Vol.12(10) | e5098 | DOI: https://doi.org/10.25248/reas.e5098.2020 Página 6 de 10 


\section{DISCUSSÃO}

A estratégia de profilaxia pós-exposição, oferecida pelo SUS no Brasil, tem indicações precisas para situações onde houve importante risco de contaminação, são elas: violência sexual, prática sexual vaginal e/ou anal com parceiro sabidamente soropositivo ou cuja sorologia seja desconhecida e de alto risco. A janela de oportunidade para administração da PEP compreende o período de até $72 \mathrm{~h}$ após o evento, apresentando efetividade superior quanto mais precoce (BRASIL, 2019). Ademais, outras infecções sexualmente transmissíveis devem ser inclusas no protocolo, abrangendo tratamento para vírus causadores de hepatites, clamídia e gonococo, bem como exame de gravidez para mulheres em idade reprodutiva.

O sistema nacional de saúde brasileiro tem como princípios fundamentais o acesso universal gratuito e equitativo à saúde, o que deveria se aplicar inclusive à estratégia da PEP. Contudo, altos níveis de estigma se correlacionam diretamente com maiores chances de necessidades de prevenção não atendidas e, assim, menor uso de serviços de testagem. Tal realidade contribui para provável subnotificação dos casos, perpetuando a cadeia de transmissão. É relevante, assim, também que novos estudos específicos sejam desenvolvidos sobre a utilização da PEP no Brasil por grupos específicos, como a população trans e profissionais do sexo, avaliando integralmente a demanda da PEP (LANDMANN-SZWARCWALD C, et al., 2016).

Segundo Hoagland B, et al. (2017), estudos sobre o tema demonstram que a educação da população sobre a disponibilidade e os efeitos benéficos da PEP promovem uma tendência de que mais pessoas se mostrem dispostas a utilizá-la em caso de exposições de risco. Ademais, reforça ainda que possa haver um efeito de motivação para mudanças comportamentais. Em consonância com tendências de saúde no geral, o déficit da informação é ainda mais grave em situações de vulnerabilidade socioeconômica.

Além das transformações nesses contextos, o comportamento sexual entre os jovens também é motivo de preocupação crescente, já que pode haver uma minimização da gravidade da patologia e encorajamento a práticas sexuais desprotegidas. Descobriu-se ainda, a partir da pesquisa, um slogan usado pelos jovens: "AIDS já não me assusta mais". Compreender este tipo de atitude requer entender o novo cenário para a AIDS criado pelos antirretrovirais, diferente das gerações marcadas pela emergência do vírus no passado. Assim sendo, as iniciativas de intervenções preventivas se aplicam cada vez mais aos cenários atuais (KERR LRFS, et al., 2017).

De acordo com Méthy N, et al. (2015), embora a prevalência do HIV aumente com o aumento da idade devido à prevalência cumulativa e à melhora da sobrevida, a incidência muito maior em 2016 é particularmente notável. As novas infecções vêm crescendo não só no Brasil, mas os Centros de Controle de Doenças dos EUA relataram que os jovens de 13 a 24 anos nos EUA respondem por $20 \%$ de todos os novos diagnósticos de HIV, $81 \%$ dos que ocorrem entre homens gays e bissexuais. Os jovens apresentam particularidades agravantes, por se submeterem a menos testes, a frequência do uso de preservativos ser menor, o consumo de bebidas alcoólicas e/ou drogas concomitantes às práticas sexuais e uma média de 4 ou mais parceiros durante a vida sexual. A associação entre tais fatores potencializa o risco de aquisição do vírus, alimentando a cadeia de transmissão.

Outros estudos, como relatado por Wall KM, et al. (2013), confirmam essas diferenças entre HSH mais jovens e mais velhos. Analisando a partir de uma perspectiva geracional, na França, gerações mais jovens de HSH são mais propensas a ter seu primeiro sexo com um homem em comparação com a geração mais velha de $\mathrm{HSH}$, que eram jovens na década de 1980. Relatos de sexo oral e anal e frequência de sexo foram mais baixos para as gerações mais velhas de $\mathrm{HSH}$, mas são mais altos entre as gerações mais jovens de hoje. Curiosamente, observam-se semelhanças nos EUA com esse aumento da frequência sexual em jovens $\mathrm{HSH}$ e heterossexuais.

O sucesso da PEP pode ser comprometido por diversos fatores, entre eles: alta taxa de não adesão e abandono, que são especialmente altas entre adolescentes e também entre aqueles que sofreram violência sexual. Estudos recentes atribuem ao aconselhamento um papel essencial para aumento dos índices de adesão, enquanto a ocorrência de efeitos adversos contribuiu para o abandono da medicação, sendo piores 
naqueles submetidos à terapia com três drogas comparativamente aos esquemas com apenas duas (CHACKO L et al., 2014).

De acordo com Bogoch II, et al. (2014), a redução da efetividade da PEP foi associada às elevadas taxas de não adesão e continuidade das medicações. Em uma metanálise que avaliou dados referentes a aproximadamente 3,6 mil pacientes incluídos em 17 estudos, as taxas de adesão variaram entre 49 e 92\%, com uma medida síntese de $69 \%$, considerando o seguimento de 28 dias. Os eventos adversos foram os maiores responsáveis pela interrupção, porém foram relatados também casos de usuários que julgaram como baixo o risco de exposição ou que consideraram o número de dias da TARV suficientes, ou, ainda, aqueles que simplesmente perderam o interesse em cumprir o seguimento das drogas prescritas.

Ao buscar inserir a PEP na prática clínica, alguns obstáculos se impõem, desde o comportamento dos pacientes à articulação das redes de saúde. Apesar de mais de $90 \%$ dos entrevistados reconhecerem a importância do uso do preservativo como ferramenta de prevenção ao HIV, a maioria (57\%) revelou não fazer uso do mesmo em todas as relações (COHEN MS, et al., 2011).

O sistema de saúde, nos âmbitos estadual e municipal, também demonstrou dificuldade em integrar o protocolo proposto pelo Ministério da Saúde. A oferta da PEP nos serviços de emergência e ambulatórios é ainda pouco difundida, em parte pela limitação de profissionais de saúde que a consideram uma forma de incitação ao não-uso dos métodos de barreira (MAKSUD I, et al., 2015; BRASIL, 2019).

O tratamento como prevenção (TcP) é apontado como a efetiva para controle da replicação visto que reduz a transmissibilidade do HIV em 96\% (FERNANDEZ SB, et al., 2013). A análise de uma coorte de 17 mil pessoas não infectadas, observada durante 7 anos na África do Sul, mostrou que para cada $1 \%$ de aumento das taxas de cobertura de TARV houve um declínio de 1,4\% no risco de infecção na população. Em 8 anos, um programa de TARV com $80 \%$ de cobertura e retenção de $85 \%$ destes no seguimento clínico reduziria entre 35 e 54\% as taxas de incidência (ROLAND ME, et al., 2011; TANSER F, et al., 2013).

O conhecimento da PEP sexual é baixo entre potenciais usuários e profissionais de saúde, mesmo com toda sua eficácia. Em um estudo nos EUA, 59,7\% dos clínicos referiram conhecer a PEP sexual, mas 39,3\% nunca havia prescrito tal método. Além disso, há pessoas que sonegam ou exercem o direito de não iniciar, motivadas pelo medo de ter o vírus e o diagnóstico traduzindo em redução das relações sexuais e aumento de práticas seguras, por vezes ao receio dos benefícios em contrapartida dos efeitos adversos e encargos do tratamento (FERNANDEZ SB, et al., 2013).

$\mathrm{Na}$ Espanha, entre pessoas que procuraram o teste anti-HIV, apenas $22 \%$ conheciam a PEP sexual e $2 \%$ já utilizaram (SCHECHTER M, et al., 2004). Entre os homossexuais o método também foi baixo. As razões foram semelhantes ao trabalho nos EUA, sendo as limitações inerentes às características individuais (percepção de não haver risco e receio de eventos adversos), dos serviços de saúde (poucos pontos de oferta e tempo de atendimento) e do próprio método (limite de 72 horas para o início da profilaxia) (ROLAND ME, et al., 2011).

Segundo o Programa Conjunto das Nações Unidas sobre HIV/AIDS (AIDS), seria viável a eliminação da doença até 2030 caso as seguintes medidas propostas fossem alcançadas: políticas públicas garantissem que $90 \%$ dos infectados tivessem conhecimento do diagnóstico e, desses, $81 \%$ tivessem carga viral indetectável no plasma. Diante de tal perspectiva, o Brasil adaptou protocolos, oferecendo início da TARV imediatamente após o diagnóstico, ampliando a perspectiva de sucesso individual e coletivo nessa janela de oportunidade. Contudo, há modelos matemáticos contrários a essa meta, que poderia ser limitada por fatores como aumento da prática sexual desprotegida, surgimento de novos métodos preventivos e rastreamento adequado insuficiente para suprir uma proporção elevada de pessoas soropositivas desconhecidas até então (GRANJEIRO A, et al., 2015).

Segundo Kuchenbecker $R$ (2015), selecionando os maiores ensaios clínicos randomizados sobre estratégias de prevenção anti-HIV, três merecem destaque: os estudos RESPECT, RESPECT 2 e AWARE, cujos resultados foram discordantes acerca do benefício do aconselhamento vinculado à testagem anti-HIV. 
No estudo RESPECT, paciente que foram orientados e receberam informações educativas sobre medidas de prevenção tiveram menor frequência de IST e HIV em 12 meses, além de uso de preservativos com maior frequência quando comparados ao grupo em que não houve intervenção, demonstrando um impacto positivo.

Os resultados estimados pelo estudo para indivíduos submetidos a aconselhamento variaram entre 32 (IC95\% 19 - 91) (aconselhamento ampliado) e 38 (IC95\% 19 - 527) (aconselhamento breve). Neste estudo não houve inclusão de homens que praticam sexo com homens (HSH) e não havia teste rápido anti-HIV disponível. Já no estudo RESPECT 2, os indivíduos foram randomizados para receber aconselhamento e teste rápido anti-HIV em uma única consulta ou testagem com teste anti-HIV padrão realizado em duas visitas. A incidência cumulativa de DST/HIV em 12 meses foi maior no grupo que recebeu teste rápido, comparativamente ao grupo controle, embora sem significância estatística: RR 1,11 (IC95\% 0,96 - 1,29; $p=$ 0,15) (KUCHENBECKER R, 2015).

Os achados de no intervalo de seis meses do estudo (curto prazo) sugerem que as intervenções baseadas em teste rápido apresentaram efetividade inferior em relação à ocorrência de IST/HIV, comparativamente ao aconselhamento-padrão. O estudo AWARE analisou o impacto de estratégias de aconselhamento curtas, no momento da realização do teste rápido anti-HIV, comparativamente a informações fornecidas exclusivamente para a realização do teste propriamente dito. Como resultado, não houve diferença na incidência de IST/HIV após seis meses de seguimento (KUCHENBECKER R, 2015).

As intervenções para aumentar a adesão têm resultados controversos:457 usuários de PEP da Califórnia (EUA) passaram por sessões de aconselhamento para promover a adesão, mas não resultaram em maior taxa de cumprimento do tratamento. No caso do TcP, a experiência de países de alta renda recomenda cautela. Em tais contextos, a cobertura de testagem e tratamento não foi acompanhada por uma redução da epidemia; situação similar à brasileira, em que valores elevados de incidência e mortalidade coexistem com taxas acima de $75 \%$ de conhecimento da positividade e do acesso universal à TARV. Assim, embora o maior efeito do TcP sobre a epidemia esteja relacionado à melhora da continuidade do cuidado, especialmente da adesão ao tratamento, outras estratégias preventivas para pessoas não infectadas são cruciais (ROLAND ME, et al., 2011).

\section{CONCLUSÃO}

A PEP foi consolidada, portanto, como uma estratégia eficaz ao intervir no curso da transmissão do HIV, principalmente quando comparada a medidas comportamentais. Aplica-se em situações de exposição sexual de risco, compartilhamento de seringas, exposição ocupacional e associa-se risco de transmissão do HIV, influenciado pela carga viral, integridade da mucosa, presença de traumatismos, entre outros. A oferta da PEP ainda é complexa graças às informações limitadas acerca dos benefícios e percepção realística dos riscos do HIV, limitando a procura em tempo oportuno, adesão e comportamentos. Contudo, o perfil de maior prevalência é o de homens que fazem sexo com homens e usuários de drogas injetáveis, corroborando os dados da literatura.

\section{REFERÊNCIAS}

1. BAGGALEY R, et al. Young key populations and HIV: a special emphasis and consideration in the new WHO Consolidated Guidelines on HIV Prevention, Diagnosis, Treatment and Care for Key Populations. J Int AIDS Society. 2015;18(2Suppl 1):19438.

2. BENN P, et al. UK guideline for the use of post-exposure prophylaxis for HIV following sexual exposure. Int J STD AIDS 2011; 22:695-708.

3. BOGOCH II, et al. Patient attrition between the emergency department and clinic among individuals presenting for HIV nonoccupational postexposure prophylaxis. Clinlnfect Dis 2014; 58(11): 1618-24.

4. BRASIL. Ministério da Saúde. Secretaria de Vigilância em Saúde. Departamento de DST, Aids e Hepatites Virais. Boletim Epidemiológico HIV/Aids. Brasília: Ministério da Saúde, 2019.

5. CAMPBELL TB, et al. Efficacy and safety of three antiretroviral regimens for initial treatment of HIV-1: a randomized clinical trial in diverse multinational settings. PLoS medicine 2012; 9(8). 
6. CHACKO L, et al. Adherence to HIV post-exposure prophylaxis in victims of sexual assault: a systematic review and meta-analysis. Sexually transmitted infections. 2011; 88(5):335-41.

7. COHEN MS, et al. HPTN 052 Study Team. Prevention of HIV-1 infection with early antiretroviral therapy. N Engl J Med 2011; 365(6): 493-505

8. FERNÁNDEZ-BALBUENA S, et al. Madrid Rapid HIV Testing Group. Awareness and use of nonoccupational HIV post-exposure prophylaxis among people receiving rapid HIV testing in Spain. HIV Med 2013; 14(4): 252-7.

9. GRANEIROL A, et al. O efeito dos métodos preventivos na redução do risco de infecção pelo HIV nas relações sexuais e seu potencial de impacto em âmbito populacional: uma revisão da literatura. Rev bras. Epidemiol. Vol.18 supl.1 São Paulo Septembro 2015.

10. HOAGLAND B, et al. Awareness and willingness to use pre-exposure prophylaxis (PrEP) among men who have sex with men and transgender women in Brazil. AIDS Behav 2017; 21:1278-87.

11. LANDMANN-SZWARCWALD C, MACINKO J. A panorama of health inequalities in Brazil. Int J Equity Health 2016 ; 15:174.

12. KERR LRFS, et al. HIV/AIDS among men who have sex with men in Brazil [HIV/AIDS em homens que fazem sexo com homens]. Paper presented at: X Congresso de Epidemiologia da ABRASCO 2017; Florianópolis - SC.

13. KUCHENBECKER R. Qual é o benefício das intervenções biomédicas e comportamentais na prevenção da transmissão do HIV? Rev. bras. epidemiol. vol.18 supl.1 São Paulo Sept. 2015

14. MAKSUD I, et al. Tecnologias de Prevenção do HIV e desafios para os serviços de saúde. Rev. bras. epidemiol. 2015 Set.; 18(Suppl 1): 104-119.

15. MARINS JRP, et al. Dramatic improvement in survival among adult Brazilian AIDS patients. AIDS. 2003;17(11):16751682.

16. MAYER KH, et al. Raltegravir, tenofovir DF, and emtricitabine for postexposure prophylaxis to prevent the sexual transmission of HIV: safety, tolerability, and adherence. Journal of acquired immune deficiencys yndromes. 2012; 59(4):354-9.

17. MÉTHY N, et al. Sexual behaviours of homosexual and bisexual men in France: a generational approach. PloS One 2015;10:e0123151.

18. OTTEN RA, et al. Efficacy of postexposure prophylaxis after intravaginal exposure of pig-tailed macaques to a humanderived retrovirus (human immunodeficiency virus type 2). Journal ofvirology. 2000; 74(20):9771-5.

19. ROLAND ME, et al. A randomized noninferiority trial of standard versus enhanced risk reduction and adherence counseling for individuals receiving postexposure prophylaxis following sexual exposures to HIV. Clinlnfect Dis 2011; 53(1): 76-83.

20. SCHECHTER, et al. Behavioral Impact, Acceptability, and HIV Incidence among Homosexual Men with Access to Postexposure Chemoprophylaxis for HIV. Journal of Acquired Immune Deficiency Syndromes; 2004v. 35, n. 5, p. 519525.

21. TANSER F, et al. High coverage of ART associated with decline in risk of HIV acquisition in rural kwazulu-natal, South Africa. Science 2013; 339(6122): 966-71.

22. WALL KM, et al. Frequency of sexual activity with most recent male partner among young, Internet-using men who have sex with men in the United States. J Homosex 2013;60:1520-38.

23. WORLD HEALTH ORGANIZATION. Guidelines on Post-Exposure Prophylaxis for Hiv and the Use of Co-Trimoxazole Prophylaxis for Hiv-Related Infections Among Adults, Adolescents and Children: Recommendations for a Public Health Approach. Recommendations for a public health approach December supplement to the consolidated ARVs guidelines WHO Geneva, n. December, p. 52, 2014. 